\title{
Studying viable free markets in Peer-to-Peer file exchange applications without Altruistic Agents
}

\author{
David Cabanillas ${ }^{1}$ and Steven Willmott ${ }^{1}$ \\ Technical University of Catalonia, \\ Software department, \\ Campus Nord, Omega building \\ Jordi Girona Salgado, 1-3 \\ Barcelona (08034), Spain \\ \{dconrado, steve\}@lsi.upc.edu
}

\begin{abstract}
File sharing networks are among the most popular applications of Peer-to-Peer (P2P) technology to date [3] and have been widely studied in terms of the performance, behavior, topology and other properties. A persistent theme throughout this research has been the evidence that many $\mathrm{P} 2 \mathrm{P}$ file sharing systems rely on the presence of altruistic users, who provide files, network capacity or some other goods without obvious personal gain to work and are potentially damaged by the presence of too many free-riders (users who consume resources but do not provide to others in return). In this paper we will explore the use of simple market mechanisms for P2P file sharing which function without the need of altruistic users considering the conditions under which such markets may be viable.
\end{abstract}

\section{Introduction}

Many P2P file sharing systems are known to rely heavily on the presence of altruistic users which act as sources for content which benefits others but not necessarily themselves [11] ${ }^{1}$. But experiences with P2P file sharing systems confirms that large resources owners are not always altruistic [2]. Economic market based systems have been proposed and in some trial systems adopted [4], widely as a regulatory mechanism to provide incentives for users to provide content/resources to a system rather than relying on the altruism to others. Systems such as Karma [7] and MojoNation ${ }^{2}$ are well known for introducing "virtual currency" based markets in order to facilitate exchange. Systems based on reputation [16], ranking [8], or other means have also been suggested.

\footnotetext{
${ }^{1}$ In some P2P system a non-negligible percentage of peers were proven to be altruistic. In Gnutella for example, $1 \%$ of peers served about $37 \%$ of the total file shared [1].

2 MojoNation has ceased operations, although information is still online: web.archive.org/web/*/mojonation.net/*.
} 
Much of the analysis of such systems ([9], [13]) however have focused on free riders - actors who take more than their fair share of the benefits or do not shoulder their fair share of the costs of their use of a resource - and how to eradicate them. The danger of the presence of too many free-riders will reduce or force to zero the number of altruists in the population - thus stopping a system from functioning. In this context an additional question arises: can a market-based system for P2P file exchange function at all without the presence of altruistic agents? and if so what conditions are necessary for it to function? It seems intuitive that the answer to the first question should be yes since digital content can arguably been seen as a good like any other. However, as it is argued in this paper there are a number of pitfalls in implementing a functioning market system and have been studied the conditions under which a file exchange market mechanism based on a "virtual currency" such as those tried in Karma and MojoNation can facilitate viable file-exchange. In general, P2P systems have two ways to encourage users to contribute to a community:

- By making users believe the idea that they are part of the community and stimulating their altruism toward the system.

- Providing a model by means of norms or rules that stimulates the users to contribute by providing a direct reward for contributions - in other words offering a return for contributions.

This paper is organized as follows. Section 2 describes the environment. Section 3 characterizes different types of markets. In section 4 are analyzed experimental results for different market configurations. Finally section 5 provides conclusions and outlook.

\section{Token based markets for P2P file sharing environments}

File-sharing applications provide the interchanges of content between users. Specifically, users typically have in their possession a certain amount of content but they would like to obtain other files they currently do not possess. Other users, in turn may wish to access the content a user may have. In an ideal world, a user would like to obtain all the content of interest to him/herself without incurring any infrastructure costs (note that in certain systems costs for content itself may apply - these are not considered here). Other members of the community however have a similar aim and given that there are inevitably some infrastructure costs incurred from providing content files to others, such as bandwidth, continual connectivity etc. the question arises as how should these costs be shared between participants?

Given the assumption that no agent in the world is willing to altruistically incur costs simply in support of the community, as in human economic systems, a balance therefore needs to be struck between a member of the community providing content and their ability to download content. A powerful mechanism to achieve this is the use of a concrete means of transferable value which can be earned by providing content and spent by downloading it. 


\subsection{Model description}

This section introduces a simple and generic market model for file interchange based on a synthetic currency (tokens) which act like a means of transferable value. Elements present in the system, are: the agents which participate, denoted by $A=\left\{a_{1}, \ldots, a_{r}\right\}$, a set of files $F=\left\{f_{1}, \ldots, f_{s}\right\}$ and a set of categories each of which groups a set of files by similar theme $C=\left\{c_{1}, \ldots, c_{t}\right\}$. In any given scenario, the three main elements for the description of model are: the initial and targeted distribution of content, the monetary system and, finally, the behavior of the members. With respect to monetary system, the financial exchange rules imposed on members of the system by the system and agent behavior strategies for purchase/sale of content and interaction with other agents, a system can be controlled according to two dimensions: 1) manipulation of market rules in order to increase/optimize exchange efficiency between agents (defined in point Monetary system) and 2) manipulation of individual agent strategies in the world such as trigger rules for when Agents do/do not make content available, defined in point Agent behavior.

Content distribution: In order to simulate content exchange, we model a random initial distribution of content in the system [15]:

- To each category, the model assigns a popularity ranking, using a Zipf ${ }^{3}$ probability distribution:

$$
p(c)=\frac{\frac{1}{c}}{\sum_{i=0}^{t} \frac{1}{i}}
$$

where $t$ is the number of categories.

- Each agent in the system has a minimum number of categories with which it is associated and an interest level in each category $\left(c^{\prime} \in C_{i}\right)$ is assigned to them w.r.t. $i l$ is determined for each agent following a random distribution. Finally, an agent $i$ that has an interest level $i l$ in each category $c$, is denoted by $p(c \mid i)$, that is drawn from a Zipf probability distribution s.t.:

$$
p(c \mid i)=\frac{i l_{c}^{i}}{\sum_{c^{\prime} \in C_{i}} i l_{c^{\prime}}^{i}}
$$

- Each category has an assigned number of files $p(c) F$, where $\mathrm{p}(\mathrm{c})$ is the fraction of files in the category $c$ and $F$ the total number of files in the system.

- Each agent has assigned a quantity of files $f_{c, r}$, where $c$ represents the category and $r$ the ranking of popularity of the file. For initial conditions, the system follows a uniformly random distribution, which assigns only files of categories where the agent has interest. With respect to the popularity of the new content, will also follow a Zipf probability distribution.

\footnotetext{
${ }^{3}$ It has been observed in [10] that many document storage systems exhibit Zipf distribution on the popularity of his documents.
} 
- Finally, the system assigns popularity to each file within a category, given by the Zipf probability distribution:

$$
p\left(f_{c, r} \mid c\right)=\frac{\frac{1}{r}}{\sum_{i=0}^{F_{c}} \frac{1}{i}}
$$

where $F_{c}$ is the number of different files in the category $c$.

These assignments, categories etc. form the basis for driving desire for content and later satisfaction generated by possession of content in the system (depicted in figure ).

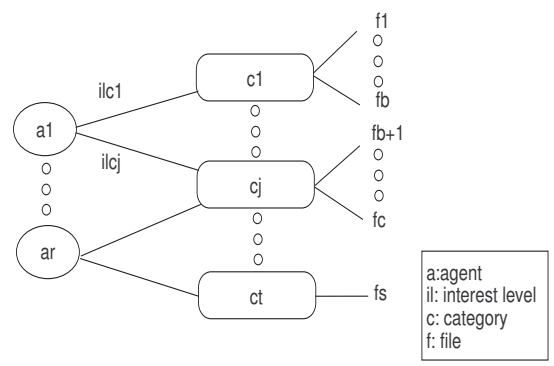

Fig. 1. Content distribution

Monetary system: A monetary system secures the proper functioning of money by regulating economic agents, transaction types, and money supply. In the model, we have agents which wish to obtain files from the market. ${ }^{4}$ Two types of transactions are possible in the system. The first one is to obtain some content; the other is to supply some content. The first agents will have average value of all cost per file. New members only arrange a minimum quantity of tokens to buy new set of files, so it forces them to offer its contents. Also, files are assigned when starting the system in a proportional way i.e. if the system has $n$ files per category and a category has $m$ agents, the agent of this category will has $n / m$ files.

The model is composed by two markets. The inner market models the mechanisms created in order to facilitate interchange of files. This inner market is embedded in an outer market which represents the real world in which users live/work and it is seen as a proxy for their individual satisfaction. The two markets are set up to use two separate currencies: in the inner market agents have IMT (inner-market-tokens) which represents the virtual currency as might be used in a system such as MojoNation and the outer market transactions are

\footnotetext{
${ }^{4}$ In the model presented here, the notion of satisfaction $B$ is used as quality measure which reflects how user's content is - increasing when desirable content is obtained, decreasing when resources are used to serve files.
} 
made using OMT (outer-market-tokens) which represents a model of satisfaction of the user either in abstract terms or (for example) in terms of a real world currency such as US dollars, Euros or Japanese Yen. The reason for using two currencies in the model is in fact to capture the use of just virtual one. The OMT models a real world currency such as the Dollar or the Euro - and the second currency, the virtual one being studied; it is created specifically for the application in question. When a consumer agent wishes to purchase content, they pay using IMT and the provider agent receives these tokens which it can barter in the future. In terms of OMT, the provider has a cost to offer the content and the consumer got a quantity of $\mathrm{OMT}^{5}$ (it is depicted in figure 2). If an agent in the system has less IMT than necessary for a particular piece of content, a purchase cannot be made and OMT will not be improved.

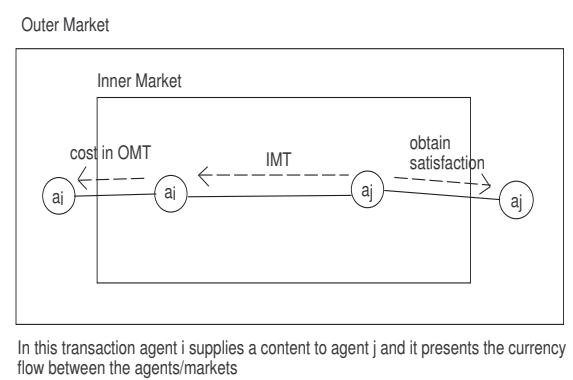

Fig. 2. Interaction between the two markets

Although real currencies could also be used in the file-sharing market (in place of IMT) this is generally not an attractive solution since real financial transactions generally incur real transactions costs and raise taxation issues.

Agent behavior: In order to describe agent behavior, in first place we establish a set of basic rules:

- Rule 1: An agent $a_{b}$ will never buy a file $f$, if $a_{b}$ is already its owner.

- Rule 2: If something costs more tokens than an agent $a_{b}$ has, $a_{b}$ cannot buy it.

- Rule $3^{6}$ : If an agent $a_{b}$ has enough tokens or it is not interested in any content, $a_{b}$ will not offer its content.

All agents in the system have an associated quantity of IMT $Q_{i m t}$ - in the inner file market and a quantity of OMT $Q_{o m t}$ - in the outer general market. The objective $O$ for all agents is maximum satisfaction $B$.

\footnotetext{
${ }^{5}$ In terms of satisfaction, agents in the system aim to maximize OMT since this value represents the satisfaction attained by agents in the system.

6 This relevant rule, relates agent behavior and the market. The main objective for all agents is to gain maximum satisfaction and not to obtain a large quantity of tokens.
} 


$$
\forall_{i \in \text { system }} O\left(a_{i}\right)=\max B
$$

$\digamma$ represents a quantity of files that an individual might desire (i.e. files that an agent has some interest). $\mathrm{B}(\digamma)$ is the utility (satisfaction) gained by that individual obtaining this quantity $\digamma$.

$$
B(\digamma)=\sum_{i=0}^{b} B\left(f_{i}\right)
$$

We model an individual's choice of $\digamma$ by assuming the consumer chooses $\digamma$ to maximize $\mathrm{B}(\digamma)$ and not by other human social instincts. This means that the agents in the model are focused on obtaining the maximum number of files that they desire rather than anything else.

$$
\digamma_{0}=\operatorname{argmax}_{\digamma} B(\digamma)
$$

holds if and only if $\digamma_{0}$ is the unique value of $\digamma$ for which $B(\digamma)$ is maximized.

Download of the desired contents is not free however, since there is some cost associated with provision of content by the potential provider agent. A content provider may then levy a fee for the content in terms of OMT, an agent seeking content must itself seek to obtain IMT if it does not already have them, creating an economic cycle. This cycle means that when paying for content, we describe $O$ as:

$$
O\left(a_{i}\right)=\max \left(\alpha \digamma_{0}-\beta Q_{O M T}\right)
$$

Once we have a clear idea about the objective followed by community members we should describe how these members can achieve it by means of their strategies. The strategy $S_{i}$ of agent $i$ is formed by a set of actions that it can follow in each step, trying to achieve agent's $O$. The available actions $A C$ in the system are:

$A C_{s}$ Agents choose to offer or withdraw from offer content to/from the system (and for each download will incur a fixed cost in real world resources in IMT and gain a fixed sum of OMT).

$A C_{d}$ Agents choose to download / not download available content, in the case of each download using up OMT but generating increased satisfaction measure by their desire for the file - converted into a monetary value in terms of IMT.

With respect to outer market, agents desire to spend minimum quantity of OMT and to achieve the maximum possible satisfaction which would correspond to obtaining all the files in the categories associated with the Agent ${ }^{7}$. With respect to the inner market, agents only offer content if they are interested in a content and they do not have enough IMT to buy it.

\section{Types of Market Scenario}

A market mechanism provides a powerful mechanism to regulate exchange between members of a community, in which each one of the members of the community wishes to maximize its utility [14]. A natural step is to create market

\footnotetext{
${ }^{7}$ In the model, 1 unit OMT is equal at 1 unit of satisfaction.
} 
places which use a type of artificial currency in order to simulate transferable value between users in a system - and hence facilitate exchange. As it is shown in this section however, there are pitfalls to doing this. In particular the types of markets envisaged include: Time limited markets, content limited markets, and time and content unlimited markets. The model for file interchange, described in [6], has three main elements that specify our system are content distribution, monetary system, and agent behavior. The most relevant aspects to look on as is that the model are:

- The model is composed by two markets. The inner market model used to study the application and the outer market model which models a real world currency.

- Agents select its strategy (offer/download content) conditioned to the quantity of tokens that they have/have not (thresholds).

\subsection{Time limited markets}

In this case, the number of interactions in a given market place is limited (time limited). Concretely, this means that in a time the system will cease functioning (for example if all files are exchanged, a certain deadline passes or after some signal is given). In a time unlimited market, members cooperate with the objective of getting a benefit in a long term future ${ }^{8}$. However, when the time is limited, the hope of a future benefit is not apparent because members know that in a concrete time the game will finish.

To comprehend the effect of this given that players know that a game has exactly $n$ rounds. Then, no matter which round has been reached (say $n-1$ ) the agent is aware that the currency used in the inner market will no longer be useful after the end of the game. Hence no agent will offer content in the last round (round $n$ ). Subsequently this also means that the currency is no use not only after the end of the game but also not in the last round. Similarly no agent will offer content in round n-1 and so forth. By repeating this argument many times, rational agents would deduce that they should not offer content at all (unless their motivation changes because someone else offers something they want). In a simulation where an agent can chose between two strategies, the only difference between the two strategies $\left(s_{1}, s_{2}\right)$ and $\left(s_{1}^{\prime}, s_{2}\right)$ is that in the period $t$ the first strategy chooses $C$ (cooperate - offer content) and the second strategy chooses $D$ (defect - not offer content). Until the end $T$ of all iterations the benefits of choosing the strategy $\left(s_{1}^{\prime}, s_{2}\right)$ will be greater than $\left(s_{1}, s_{2}\right)$. This concept is clearly analogous in the well known game theory known as the Prisoner's Dilemma (PD) [5] result for games of known duration. ${ }^{9}$ The conflict between the individual and collective interests is expressed in this game, which has implications in real life in areas like the policy, society, economy. Concretely the relation is with a subset of $\mathrm{PD}$, named $\mathrm{PD}$ with finite repetitions.

\footnotetext{
8 The shadow of the future [5].

${ }^{9} \mathrm{PD}$ rules are explained in detail in [12].
} 


\subsection{Content limited markets}

This hypothesis considers that the content is limited even if time were unlimited. In such world the number of total different content items is finite and unchanging. In an ideal world all members in the market should obtain all contents that they want. If agents are aware of this fact, this goal will not be achieved. When an agent obtains all the content that it desires (satisfied agent) it is conscious of the fact that it has all it may want so a rational agent would cease offering content. The reason is similar to that in the previous case: the agent will, in the future, not derive benefit from the inner-market tokens (IMT) obtained. This fact entails that other non-satisfied agents may not obtain all the content they desire if some of it is held by satisfied agents. The tokens have value for an agent if they can be exchanged for something desirable. Once it is known that there is no more new content to obtain, the value of tokens tends to zero. In turn, this causes the agent to become resistant to offering content before all possible useful exchange have been made. Only altruists would continue once they had obtained everything they needed.

\subsection{Time and content limited markets}

Under these restrictions, a market has little hope of functioning. An interesting example of this can be seen in exemplify by Clive Thompson in his article "Not With a Bang but a Whimper" about the game Asheron's Call $2^{10}$, an online game scheduled to cease functioning in December 2005. Characters in the game may pick up items such as tools, armor and weapons at once within a container and they can trade these items with other players. When the game was flowering the characters used to sell their items but when the game shut down was first announced, the majority of players left the game. This happens because without a sense of future capitalism ends. In other words there's no demand in a condemned world.

\subsection{Time and content unlimited markets}

In the previous section it was argued analytically that markets limited in time or/and content function sub-optimally, if at all. In this section, we move on to the case of behavior of the market without these limitations. With respect to the cost of offering a piece of content versus the satisfaction that someone can obtain from obtaining outer-market tokens (OMT), we have the following alternatives:

A. If the cost of offering is less than the benefit obtained: In this case, agents have interest in offering their contents because they can obtain benefit of it in return - a benefit that in the future the agent can re-invest.

B. If the cost of offering is equal to the benefit obtained: In this case, no net benefit is generated through offering content on average.

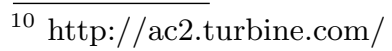


C. If the cost of offering is greater than the benefit obtained: In this case file exchange generates a net loss for the community over time and most likely for the individual - increasing with the number of transactions carried out.

For the three options above it is probable that A and B could function in some form (although option B only in a very limited manner), while option C appears to be unsustainable in the long run since agents in the system will all incrementally loose satisfaction.

\section{Experimental Evaluation}

In this section we describe a number of simulations which help to clarify the nature of the dynamics of a token-based P2P market under the scenarios listed in the previous section - Time and content unlimited markets.

\begin{tabular}{|c|c|c|}
\hline Symbol & Meaning & Value \\
\hline$A$ & n of agents & 200 \\
\hline$F$ & n of files & 200 \\
\hline$C$ & n of categories & 5 \\
\hline$C x A$ & n of categories x agents & 2 \\
\hline Cimt $_{f_{x}}$ & Cost per file (IMT) & 500 \\
\hline Bimt $_{f_{x}}$ & Benefit per file (IMT) & 500 \\
\hline Comt $_{f_{x}}$ & Cost per file (OMT) & Minimum Bomt $f_{x}$ \\
\hline Bomt $_{f_{x}}$ & Benefit per file (OMT) & Greater than Comt $f_{f_{x}}$ \\
\hline \multicolumn{3}{|c|}{ Table 1. Initial experiment parameters } \\
\hline \multicolumn{3}{|c}{}
\end{tabular}

Agents in the system ${ }^{11}$ do not act altruistically ${ }^{12}$ and this is concretely interpreted as a fixed rule: agents only offer content to generate IMT up to a set limit (threshold) which is the level the agent expects to be able to usefully spend on new content. Further, since an agent cannot buy content if it has less IMT. By means of these thresholds, the period where agents offer content is constrained by need. When an agent has more IMT than threshold related with the supply, no content is offered. Further, since an agent cannot buy content if it has less IMT a simple layered model emerges as shows in figure 3.

\subsection{Experimental results}

In this section we analyze the results of experiments simulating options 2 and 4 above. Different cases considered for option 2 are:

11 Table 1 describes the system settings.

12 However when the system starts to work in the initial state some agents are randomly selected and forced to offer their contents. Without this jump start, no agents would offer content intially. 


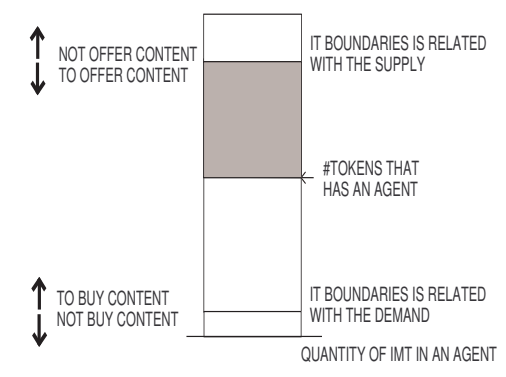

Fig. 3. Boundaries related with IMT

Simulation 1: At this case agents have a quantity of 2900 IMT, near to threshold related with the supply.

Simulation 2: At this case 2000 IMT per agent.

Simulation 3: At this case 600 IMT per agent, near to threshold related with the demand.

In option 4 , three cases are considered:

Simulation 4: Half of the members 600 IMT and the other half 2000 IMT.

Simulation 5: Half of the members 200 IMT and the other half 2000 IMT. Simulation 6: Half of the members 200 IMT and the other half 6000 IMT.

Figures 4 a) and 4 b) show the cdf of the different scenarios proposed above, in terms of quantity of files exchanged in the system and times that agents did not have enough tokens to buy contents when they would have liked to (indicating inefficiency of the market as an exchange mechanism) respectively. The first figure shows the relationship between quantity of tokens and number of files on sale. The second figure relates the threshold and quantity of times an agent in the system does not have enough IMT to buy content. Both figures show the importance of the amount of tokens that the agents in the system runs.

\subsection{Evaluation Results \& Discussion}

The simulations show the following results:

- The first observation is that at the beginning all simulations show a significant increase of activity due to different facts: initially, agents have enough money to buy at least one file; also we may think about many agents having an interest in the content offered by the rest of members.

- Simulations 1, 2 and 3 reveal that the quantity of tokens in the system modifies the behavior of the market, in terms of global number of files exchanged (at satisfaction level). Reviewing values shown in figures 4 a) and $4 \mathrm{~b}$ ), in a first glance it is shown that in simulations 2, 6 and specially 3, in many cases agents wants to buy content but they do not have enough tokens to 

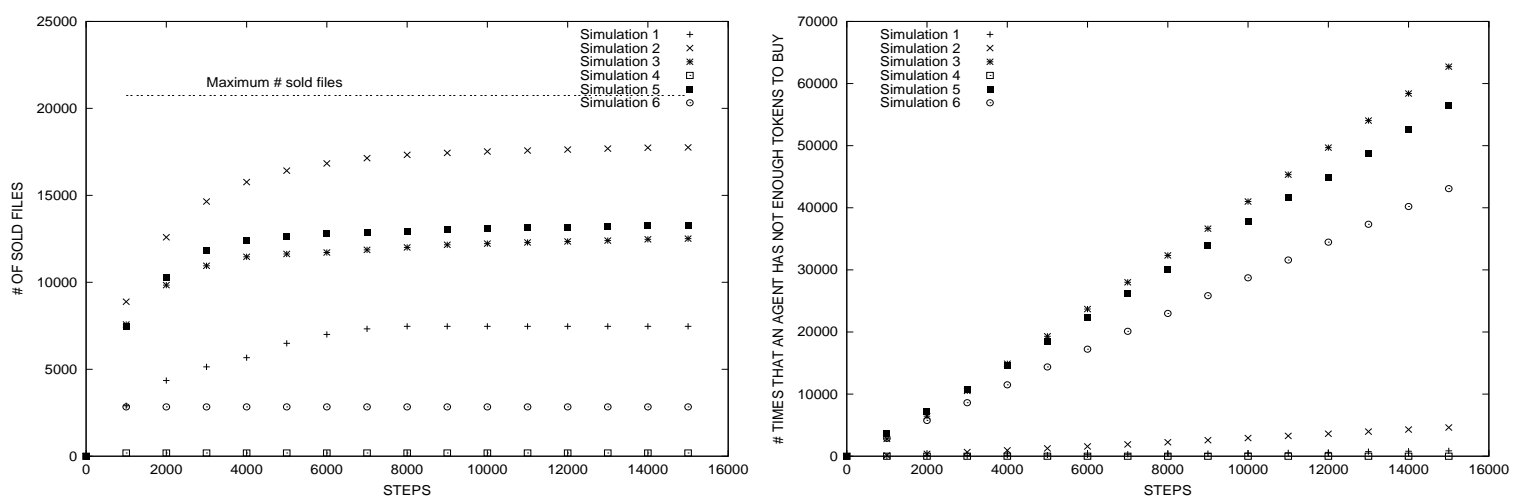

Fig. 4. a) Experimental results related with number of sold files x simulation b) Experimental results related with number of times that agents in the system does not have enough tokens

buy anything, showing that it is not a optimum market. Reviewing in detail different steps in the simulation 3 , it can be seen that the distribution of tokens is not appropriate to the right working of the system: Some agents pass boundary related to supply, so they can't offer anything; and others agents do not have enough tokens to buy content.

- Simulation 4 shows that selfish agents can actually prevent the system from working correctly. This occurs because; if an agent has more tokens than threshold supply they will not offer their content. And, in this case, the remaining agents have a number of tokens near to the threshold supply. Hence once a few files have been purchased, they also pass this quantity and cease offering files. This confirms the stability of market fails in the case of token oversupply.

- In simulation 5 , agents that have fewer tokens than the purchasing threshold can trade to move above the threshold. Limited trade becomes stable in token undersupply situations.

- In simulation 6, agents that have more tokens than the threshold supply can trade with agents that have fewer tokens. A transferring of tokens is generated from agents that have tokens to agents who have not tokens.

Throughout this paper we have discussed which market conditions which are/are not viable for P2P file sharing systems. While the restrictions discussed in this paper do not apply to all $\mathrm{P} 2 \mathrm{P}$ systems they may certainly arise in systems. Examples could include: 1) limited content a system of interchange of contents could exist specialized. In particular movie categories, 2) limited time markets in special short-term corporate promotions (where tokens loose validity after a certain date) or 3) in time/content unlimited scenarios where the balance between cost and benefit is very fine. The analysis and experiments show that:

- Markets finite in time or content are likely to fail (either because agents can reason about the eventual collapse of the token currency, or because content 
is withdraw from sharing to early once some agents gather all the files they are interest in).

- That even in markets with infinite time and content, where token based economies can function, barriers still exist to fluid interchange even if the cost/benefit of trading files is above zero.

- Money supply issues in infinite time and content markets play a large role in success/failure (as implicitly does new content supply). This mirrors real world inflation/deflation/money supply issues in a simple way which is unsurprising. However, in such limited environments, effects are more dramatic and further the existence of upper and lower bounds suggest that optimal values may exist which would need to change over time with the amount of users and content.

The first result suggests that artificial currencies would not be a good solution for time/content limited scenarios and in these cases, despite the added cost/complexity, real currency approaches may need to be used. The second two statements suggest that even in cases where virtual currency approaches could be applied, careful management of the currency in question needs to be carried out - most likely regulating the money supply over time to ensure efficient functioning.

\section{Conclusion and Outlook}

The results presented here provide a rough classification of types of token-based markets. In order to understand these phenomena in detail however, more work is needed in particular to: establish the range of conditions under which such a phenomena arise, analyze the detailed dynamics of those cases under which the system works. However, the overall aim of further work would be to explore money supply and market policy issues in order to manage the economy of the inner market to keep it in the identified functional zone. Each of the model changes considered above would likely change the visible market dynamics but the underlying results of a relatively narrow set of market conditions being viable seems likely to be stable.

\section{References}

1. Eytan Adar and Bernardo A. Huberman. Free riding on gnutella. First Monday, $5(10), 2000$.

2. K. Ranganathan adn M. Ripeanu, A. Sarin, and I. Foster. Incentive mechanisms for large collaborative resource sharing. In Proc. of the 4 th IEEE/ACM International Symposium on Cluster Computing and the Grid (CCGrid 2004), Chicago, USA, April 2004 (accepted for publication).

3. Stephanos Androutsellis-Theotokis and Diomidis Spinellis. A survey of peer-topeer content distribution technologies. ACM Comput. Surv., 36(4):335-371, 2004.

4. Panayotis Antoniadis and Costas Courcoubetis. Market models for p2p content distribution. In Gianluca Moro and Manolis Koubarakis, editors, AP2PC, volume 2530 of Lecture Notes in Computer Science, pages 138-143. Springer, 2002. 
5. Robert M. Axelrod. The Evolution of Cooperation. Basic Books, 1984.

6. David Cabanillas and Steven Willmott. Studying viable free markets in peer-topeer file exchange applications without altruistic agents. Technical Report LSI-0612-R, Department of Computer Science, University of Catalonia, March 2006.

7. Flavio D. Garcia and Jaap-Henk Hoepman. Off-line karma: A decentralized currency for peer-to-peer and grid applications. In John Ioannidis, Angelos D. Keromytis, and Moti Yung, editors, ACNS, volume 3531 of Lecture Notes in Computer Science, pages 364-377, 2005.

8. Sepandar D. Kamvar, Mario T. Schlosser, and Hector Garcia-Molina. The eigentrust algorithm for reputation management in $\mathrm{p} 2 \mathrm{p}$ networks. In $W W W$ '03: Proceedings of the 12th international conference on World Wide Web, pages 640-651, New York, NY, USA, 2003. ACM Press.

9. Sepandar D. Kamvar, Mario T. Schlosser, and Hector Garcia-Molina. Incentives for combatting freeriding on p2p networks. In Euro-Par, pages 1273-1279, 2003.

10. Robert R. Korfhage. Information storage and retrieval. John Wiley \& Sons, Inc., New York, NY, USA, 1997.

11. Nikos Ntarmos and Peter Triantafillou. Aesop: Altruism-endowed self-organizing peers. In Wee Siong Ng, Beng Chin Ooi, Aris M. Ouksel, and Claudio Sartori, editors, DBISP2P, volume 3367 of Lecture Notes in Computer Science, pages 151165. Springer, 2004.

12. William Poundstone. Prisoner's Dilemma. Doubleday, New York, NY, USA, 1993. Based On Work By-John Von Neumann.

13. J.A. Pouwelse, P. Garbacki, D.H.J. Epema, and H.J. Sips. The bittorrent p2p file-sharing system: Measurements and analysis. In 4th International Workshop on Peer-to-Peer Systems (IPTPS). LNCS (to appear), feb 2005.

14. K. Ranganathan, M. Ripeanu, A. Sarin, and I. Foster. To share or not to share' an analysis of incentives to contribute in file sharing environments. In Workshop on Economics of Peer-to-Peer Systems, Berkeley, CA, USA, 2003.

15. Mario Schlosser and Sepandar Kamvar. Simulating a p2p file-sharing network. In 1st Workshop on Semantics in Grid and P2P Networks, 2002.

16. Yao Wang and Julita Vassileva. Trust and reputation model in peer-to-peer networks. In Peer-to-Peer Computing, pages 150-. IEEE Computer Society, 2003. 